\title{
The Priming Function of In-car Audio Instruction
}

\begin{tabular}{|r|l|}
\hline Journal: & Quarterly Journal of Experimental Psychology \\
\hline Manuscript ID & QJE-STD 17-028.R2 \\
\hline Manuscript Type: & Standard Article \\
\hline Date Submitted by the Author: & n/a \\
\hline Complete List of Authors: & $\begin{array}{l}\text { Keyes, Helen; Anglia Ruskin University - Cambridge Campus, Psychology } \\
\text { Whitmore, Antony; Anglia Ruskin University - Cambridge Campus, } \\
\text { Psychology } \\
\text { Naneva, Stanislava; Anglia Ruskin University - Cambridge Campus, } \\
\text { Psychology } \\
\text { McDermott, Daragh; Anglia Ruskin University - Cambridge Campus, } \\
\text { Psychology }\end{array}$ \\
\hline Keywords: & visual priming, audio priming, multisensory priming, driving behaviour \\
\hline &
\end{tabular}




\section{The Priming Function of In-car Audio Instruction}

2

3 Helen Keyes*

4 Department of Psychology, Anglia Ruskin University, Cambridge, UK

$5 \quad *$ Corresponding Author

6 helen.keyes@anglia.ac.uk; 08451962923

7

8 Antony Whitmore

9 Department of Psychology, Anglia Ruskin University, Cambridge, UK

10 antony.whitmore@gmail.com; 07867600040

11

12 Stanislava Naneva

13 Department of Psychology, Anglia Ruskin University, Cambridge, UK

14 stanislava.naneva@student.anglia.ac.uk

15

16 Daragh McDermott

17 Department of Psychology, Anglia Ruskin University, Cambridge, UK

18 daragh.mcdermott@anglia.ac.uk; 08451965104

19 


\section{The Priming Function of In-car Audio Instruction}

21 Studies to date have focused on the priming power of visual road signs, but not

22 the priming potential of audio road scene instruction. Here, the relative priming 23 power of visual, audio and multisensory road scene instructions were assessed. In 24 a lab-based study, participants responded to target road scene turns following 25 visual, audio or multisensory road turn primes which were congruent or 26 incongruent to the primes in direction, or control primes. All types of instruction 27 (visual, audio, multisensory) were successful in priming responses to a road scene. 28 Responses to multisensory-primed targets (both audio and visual) were faster than 29 responses to either audio or visual primes alone. Incongruent audio primes did not 30 affect performance negatively in the manner of incongruent visual or multisensory 31 primes. Results suggest that audio instructions have the potential to prime drivers 32 to respond quickly and safely to their road environment. Peak performance will be 33 observed if audio and visual road instruction primes can be timed to co-occur. Keywords: Auditory priming; visual priming; multisensory priming; driver behaviour 


\section{Introduction}

43 The use of in-car navigation systems has grown exponentially during the past few decades, 44 and is expected to continue to grow (Broy, 2006). Traditionally, these systems have been 45 used to provide navigational information to drivers. While these devices have been studied 46 extensively in terms of their potential to distract drivers from the road scene (Dingus, 47 McGehee, Hulse, Jahns \& Manakkal, 1995; Srinivasan \& Jovanis, 1997; Regan, Oxley, 48 Godley \& Tingvall, 2001), or benefit drivers by effectively providing route-guidance 49 information (Barrow, 1991; Fastenmeier, Haller \& Lerner, 1994; Burns, 1997; Burnett, 50 2000), little work to date has looked at their potential to directly prime drivers to respond 51 quickly and safely to the road environment. Considering the usefulness of other cues such as 52 road signs in priming drivers to respond quickly and effectively to their road environment 53 (Crundall \& Underwood, 2001; Koyuncu \& Amado, 2008), it is important to explore the 54 potential of in-car devices to do the same.

The priming paradigm has become an important tool in measuring how a road cue or 56 device affects driver behaviour. While driving studies had previously focused on a driver's 57 ability to recall or name a road sign (Johansson \& Backlund, 1970; Milošević \& Gajić, 1986), 58 Fisher (1992) proposed that a more important measure of a road cue's effectiveness is how it 59 affects a driver's response - i.e. the extent to which it can successfully prime a driver such 60 that subsequent responses to the road environment are faster and more accurate. Indeed, 61 implicit measures of a road cue's efficacy are considered to be more useful than measures 62 examining explicit recall or description, as implicit measures are more closely tied to actual 63 modifications in driver behaviour (e.g. slowing down; Summala \& Hietamäki, 1984). On this 64 basis, numerous lab-based studies have focused on the implicit processing of road sign 65 information using a priming paradigm (e.g. Crundall \& Underwood, 2001; Charlton, 2006; 
66 Koyuncu \& Amado, 2008). Using this paradigm, a road cue is considered to be effective if its

67 presence facilitates a reaction to a road scene in terms of speed and/or accuracy.

68

69

70

71

72

73

74

75

76

77

78

79

80

81

82

83

84

85

86

87

88

89

Drivers use several cues in order to respond appropriately to the road environment. Road signs are the most frequently used visual primes in preparing drivers to respond (Koyuncu \& Amado, 2008), and they play a crucial role in road safety. Indeed, road signs indicating an upcoming intersection or curve in the road can decrease the number of crashes at these sites by as much as 30-40\% (Creasy \& Agent, 1985; Agent, Stamatiadis \& Jones, 1996). In lab-based studies, both accuracy and response speed are improved by road sign priming. Crundall and Underwood (2001) presented participants with priming images of road signs ("turn left" or "turn right") followed by images of road bends that were congruent or incongruent in direction. They report that for experienced drivers, congruent road sign priming significantly decreased response time to a subsequent road bend target, relative to incongruent or control priming (see Koyuncu \& Amado, 2008 for similar results). Similarly, Charlton (2006) demonstrated that semantic road sign priming increased accuracy and improved response times to images of hazardous road scenes. These studies demonstrate the importance of the priming function of road signs in responding to a road environment.

It is becoming increasingly clear that the safest way to present in-car navigational information to drivers is in audio rather than visual form, as in-car glancing to perform secondary tasks such as looking at a GPS device increases drivers' risk of crashing for both experienced (Klauer, Guo, Sudweeks, \& Dingus, 2010; Liang, Lee, \& Yekhshatyan, 2012) and novice drivers (Simons-Morton, Guo, Klauer, Ehsani \& Pradhan, 2014). The addition of auditory prompts can significantly improve driving performance compared to when information is presented visually on an in-car device (Walker, Alicandri, Sedney \& Roberts, 1991; Liu, 2001), and this is likely to be due to both a reduction in visual workload (Labaile, 
90 1990; Walker et al., 1991; Xie, Zhu, Guo \& Zhang, 2013) and the reduction in glance-time

91 away from the road scene (Jensen, Skov \& Thiruravichandran, 2010). In a comprehensive 92 mixed methods study, Dalton and colleagues (2013) performed the first large-scale 93 investigation of how the auditory presentation of in-vehicle navigational information can 94 affect driving performance. They report a memory advantage and a user preference for 95 auditory over visual presentation of route information. However, that study focused largely 96 on the navigational function, rather than the priming potential of audio presented in-vehicle 97 guidance - an area which has been overlooked in the literature.

The redundant signals effect (Kinchla, 1974) describes a phenomenon whereby 99 processing is speeded when information is presented in two different sensory channels 100 compared to either channel alone. Whereas a race model (e.g. Raab, 1962) predicts this gain 101 to be a result of activation of the faster of two responses, which are activated separately, 102 Miller's seminal paper (1982) demonstrated that multisensory input produces processing 103 gains that cannot be explained by separate activation of the single senses involved. Instead, 104 congruent input from different senses combines to produce a redundancy gain due to a 105 response threshold being reached more quickly with input from two sources compared to one.

106 Indeed, it has been proposed that presenting road environment cues in a multisensory 107 manner - e.g. in both visual and auditory form - should maximise their efficacy (Castro \& 108 Horberry, 2005). Multisensory cues are generally better at capturing spatial attention during 109 an attention-demanding task such as driving, compared to either visual or auditory cues alone 110 (see Spence \& Santangelo, 2009 for a review), and previous work has shown that the 111 provision of multisensory auditory and vibrotactile cues lead to faster braking times than the 112 presentation of auditory or vibrotactile cues alone (Ho, Reed \& Spence, 2007). Of particular 113 interest to driving researchers are the processes contributing to making a decision in a road 
114 environment. The coactivation model outlined by Miller (1982) specifically suggests that the 115 presentation of information in more than one modality should lead to speeded decision 116 responses. As such, the multi-modal presentation of auditory and visual priming information 117 should lead to improved driving performance in response to a road scene target compared to 118 either auditory or visual priming cues alone. A further advantage of presenting road cue 119 information aurally as well as visually is that there is less chance that a solitary visual road 120 sign cue - which may be obscured or not attended to - will be missed.

\section{Aims and rationale}

123 No research to date has directly compared the effects of visual road sign priming and auditory 124 priming on a road scene task. Considering that over $75 \%$ of drivers that use in-car 125 navigational devices utilise the auditory function (Dalton et al., 2013), of interest is whether 126 the addition of an audio prime can strengthen the priming effects traditionally observed for 127 road signs. Similar to the method used by Crundall and Underwood (2001) and Koyuncu and 128 Amado (2008), we used a lab-based priming paradigm to present participants with images of 129 road bend signs followed by semantically related or unrelated images of target road bends. 130 We modified this paradigm to include auditory primes in order to investigate the relative 131 priming power of visual and audio road turn primes. Of interest was whether conventional in132 car auditory instruction - "turn left" or "turn right" - primarily used to convey navigational 133 information, could be useful in priming drivers to respond rapidly to a road scene. Finally, we 134 proposed that the concurrent presentation of multisensory visual and auditory road cue primes 135 would produce additional benefit over the presentation of either type of prime alone.

136 In a naturalistic setting, a road turn sign will typically be viewed for $700 \mathrm{~ms}-1 \mathrm{~s}$ (Mori 137 \& Abdel-Halim, 1981). We designed our study to mirror this real-world experience closely 
138 by presenting primes for 580-882 ms. Auditory in-vehicle navigational instructions are often 139 presented $30 \mathrm{~s}$ in advance of a road event, with a reminder prompt optimally presented at 4-7 $140 \mathrm{~s}$ in advance of a road turn (Green \& George, 1995; Wu, Huang \& Wu, 2009). We are 141 interested in the potential additional priming function of these instructions if presented at 142 closer temporal proximity to the required road task. We focused here on semantic priming, 143 whereby a road sign or auditory prime is followed by a target road turn image, as this kind of 144 priming is naturally occurring in the driving experience.

147 Participants

148 Thirty-two participants (19 female) with a mean age of 29.3 years $(\mathrm{SD}=11.7)$ volunteered to 149 take part in the study. Participants were required to have a full, clean driving licence with at 150 least two years' driving experience in the UK (average length of experience $=8.6$ years, $\mathrm{SD}=$ 151 8.8). This research complied with the American Psychological Association Code of Ethics 152 and received ethical approval from the Psychology Departmental Research Ethics Panel at 153 Anglia Ruskin University. Informed consent was obtained from each participant. Participants 154 were paid $£ 7$ for taking part in the experiment.

157 Prime stimuli for the visual prime condition comprised images of standard UK road signs 158 indicating a left or right bend in the road, taken from the publically available Highway Code: 159 traffic signs publication (Department for Transport, 2007). The control stimulus for this 160 condition was created by replacing the left or right bend arrow in the road sign with "XXX" 161 using Adobe Photoshop. These stimuli were scaled to 600 X 524 pixels. Prime stimuli for the 
162 audio prime condition comprised a computer generated voice saying "turn left", "turn right" 163 or "hello" (control condition). These stimuli were created using free online text-to-speech 164 software and set to speak with a female UK voice, and were converted to mp3 files using 165 Audacity software. Prime stimuli for the multisensory prime condition comprised pairings of 166 visual and audio stimuli. These pairings were always congruent (e.g. the left bend road sign 167 was always accompanied by the audio instruction "turn left").

168 Target stimuli comprised eight images of local Cambridge road scenes (four rural, 169 four urban) taken in similar lighting conditions using a Nikon D60 camera. The road scenes 170 had a distinct road bend in either the left or right direction and were clear of people and cars. 171 Each road scene was saved in its original form and a mirror reversed form using Adobe 172 Photoshop to create 16 road scenes in total (eight left and eight right bends). These images 173 were converted to greyscale and any distractors (such as road signs visible in the scene) were 174 removed. The images were then cropped such that the centre of each road bend was central to 175 the image, and were saved at $1100 \times 884$ pixels. Images were viewed on a 17 inch screen of 176 a Dell PC. Images subtended a viewing angle of 11.66 by 12.96 degrees (primes) or 18.98 by 17723.41 degrees (targets) when viewed from a distance of approximately $70 \mathrm{~cm}$.

\section{Procedure}

180 Participants ran nine practice trials followed by six blocks of test trials. A trial comprised the 181 presentation of a prime (visual, audio, or multisensory), followed by a fixation cross for 300 $182 \mathrm{~ms}(\mathrm{SOA}=1,182 \mathrm{~ms})$, followed by the presentation of a target stimulus. The visual primes 183 and the visual element of multisensory primes were always presented for $882 \mathrm{~ms}$, whereas the 184 audio primes and audio element of multisensory primes were presented for 580 ms ("hello") $185-882 \mathrm{~ms}$ ("turn left" and "turn right"). In choosing our prime durations, we were guided on 
186 the literature which showed that road signs are typically viewed for a comparable length of 187 time (700-1,000 ms; e.g. Mori \& Abdel-Halim, 1981). While it is difficult to ensure that 188 audio primes using different word have identical durations (in this instance, the congruent 189 and incongruent audio primes had a duration of $882 \mathrm{~ms}$, and the control audio prime had a 190 duration of $580 \mathrm{~ms}$ ), to counteract against this, SOA's were held strictly at 1,182 ms for all 191 trial types. A prime was followed by a fixation cross, followed by the presentation of a target 192 stimulus. The target stimulus was an image of a road scene, presented until the participant 193 responded. Participants were required to press a button on the keyboard ("z" or "m") to 194 indicate whether the road scene depicted a left turn or a right turn, respectively. Each trial 195 was followed by an inter-trial interval (ITI) varying randomly between 700 and 1,200 ms. See 196 Figure 1 for an illustration of a single trial. Participants were instructed to respond to the road 197 scene as quickly and as accurately as possible.Trials were balanced such that each prime 198 medium type (visual, audio, multisensory) was congruent to the target road scene in direction, 199 incongruent to the road scene in direction, or a control an equal number of times. Trials were 200 presented in a randomised order within each block. Six testing blocks comprised 144 trials 201 each, resulting in 864 trials in total (3 prime types X 3 prime-target congruencies X 16 road 202 scenes X 6 repetitions each).

204 (Figure 1 goes here)

205

206 Results 


\section{RT Analyses}

208 Response times for incorrect responses were excluded from analysis and response times

209 further than 2 SD away from each participant's mean were excluded as outliers (Ratcliff, $2101993 ; 3.96 \%$ of correct responses). A 3 X 3 repeated measures ANOVA with factors of 211 Medium (Visual, Audio, Multisensory) and Prime-Target Congruency (Congruent, 212 Incongruent, Control) revealed a significant interaction between Prime-Target Congruency 213 and Medium, $F(4,124)=3.27, M S E=136.39, p<.05, \eta_{p}^{2}=.095$. Follow-up tests were 214 carried out separately for visual, audio and multisensory prime trials.

215 For trials containing both visual and audio priming information, participants 216 responded significantly faster where the prime-target information was congruent compared to 217 when this information was either incongruent, $t(31)=2.73, p<.05$, or control, $t(31)=2.69, p$ $218<.05$. There was no difference in response time to incongruent and control trials, $t(31)=$ 219 1.17, n.s. All results hold after Bonferroni correction for three comparisons.

This pattern was repeated for trials containing only visual primes: responses to 221 congruent trials were faster than responses to either incongruent, $t(31)=1.75, p<.05(p=$ 222.045 ; approaches significance following Bonferroni alpha adjustment to .017 for three 223 comparisons), or control trials, $t(31)=2.77, p<.05$ (significant following Bonferroni 224 correction for three comparisons), with no difference in reaction time between incongruent 225 and control trials, $t(31)=.06$, n.s.

226 A different pattern was observed for audio prime trials. Here, responses were once 227 more faster in response to congruent trials compared to either incongruent, $t(31)=3.48, p<$ 228.05 , or control trials, $t(31)=7.03, p<.05$. However, responses to incongruent audio prime 229 trials were significantly faster than responses to control audio prime trials, $t(31)=2.42, p<$ 
230 .05. All results hold after Bonferroni correction for three comparisons. See Figure 2 for an 231 illustration of these interaction effects.

233 (Figure 2 goes here)

235 Overall, a significant main effect of Prime-Target Congruency was observed, $F(2,62)=$ $23611.71, M S E=459.10, p<.001, \eta_{\mathrm{p}}^{2}=.274$, with follow up tests showing a straightforward 237 priming effect such that participants responded faster to congruent trials compared to either 238 incongruent trials, $t(31)=3.06, p<.005$, or control trials, $t(31)=5.26, p<.005$. There was 239 no difference in reaction time for incongruent and control trials, $t(31)=0.60$, n.s. All results 240 hold after Bonferroni correction for three comparisons. See Figure 3 for an illustration of this 241 effect.

242

243 (Figure 3 goes here)

245 A significant main effect of Medium was also observed, $F(2,62)=38.72, M S E=243.30, p<$ $246.001, \eta_{\mathrm{p}}{ }^{2}=.555$. Follow-up tests revealed that participants responded significantly faster on 247 trials where multisensory primes were presented, compared to trials where either visual 248 primes, $t(31)=6.78, p<.001$, or audio primes, $t(31)=7.71, p<.001$, were presented alone. 249 When presented on their own, participants responded faster to trials containing visual primes 250 compared to audio trials, $t(31)=3.62, p<.001$. All results hold after Bonferroni correction 251 for three comparisons. See Figure 4 for an illustration of this effect. 
253 (Figure 4 goes here)

254

255 Accuracy Analyses

256 As expected with a simple forced-choice task, accuracy performance was close to ceiling 257 (average accuracy $=98.01 \%, \mathrm{SD}=0.58 \%$ ). There was no main effect of Medium, $F(2,62)=$ $2582.70, M S E=2.31$, n.s., nor was there an interaction between Medium and Prime-Target 259 Congruency, $F(4,124)=1.48, M S E=2.47$, n.s. There was a straightforward effect of Prime260 Target Congruency, $F(2,62)=7.99, M S E=2.98, p<.01, \eta_{\mathrm{p}}^{2}=.205$, with planned 261 comparisons showing participants scoring significantly less accurately on incongruent trials 262 compared to both congruent trials, $t(31)=2.52, p<.05$, and control trials, $t(31)=3.56, p<$ 263.05 . There was no difference in accuracy between congruent and control trials, $t(31)=1.17$, 264 n.s.

\section{Discussion}

267 In a road scene task, we report strong priming effects for visual road sign primes, audio 268 instruction primes and a combination of both of these types of prime. Responses to a road 269 scene were fastest when priming information was presented concurrently using audio and 270 visual primes, compared to using either visual or audio primes alone. Finally, we report that 271 the presentation of incongruent audio information may not be detrimental to road scene 272 performance in the same way that the presentation of incongruent visual information is.

273 Audio information can be useful in providing navigational information to drivers in a 274 safe manner (Klauer et al., 2010; Liang et al., 2012; Dalton, 2013; Simons-Morton et al., 
275 2014). An aim of this study was to investigate whether audio information can also be used to 276 serve a priming function, improving reaction speed to a road scene. We report here that in-car 277 audio priming (e.g. "turn left") does indeed prompt participants to react more quickly to a 278 subsequent road scene. In demonstrating the priming function of auditory road instruction for 279 the first time, we highlight an additional potential benefit of audio in-car information 280 systems, beyond the provision of navigational information.

281 A main finding from this experiment is that drivers respond most quickly to a road 282 scene when visual and auditory prompts are presented concurrently. This was expected, 283 considering the general benefits of presenting information in a multisensory manner (e.g. 284 Spence and Santangelo, 2009), including road environment information (Castro \& Horberry, 285 2005). Novel to this study is the suggestion that - in order to be most effective - auditory 286 road environment priming prompts should be timed to coincide with visual road sign prompts 287 wherever possible. This result can be explained in terms of a redundancy gain, whereby the 288 concurrent activation of auditory and visual senses with congruent input lead to a response 289 threshold being reached more quickly compared to when the information was presented in 290 either sensory modality alone (e.g. Miller, 1982).

291 Because visual road signs elicited faster responses to road scenes than did audio 292 instruction alone, we should be cautious to avoid suggesting that audio primes should 293 supplant visual driving primes. Rather, we highlight the supplementary role that audio 294 information can play in road scene priming - bolstering the effects of visual road sign 295 priming when audio primes are timed to coincide. In-car audio instruction typically focuses on providing navigational information in a 297 timely manner. In an on-road driving experiment, drivers selected 8-9 s before a turn is 298 required as the ideal presentation time of audio navigation information (e.g. 148 meters from 
299 turn at $40 \mathrm{mph}$; Green \& George, 1995). In our experiment, the prime was presented $1.2 \mathrm{~s}$

300 prior to the required road turn. Decreasing the time between a prime and a target is likely to 301 improve its priming efficacy (e.g. Huber \& O'Reilly, 2003). However, we should be cautious 302 here and note that drivers need enough time to respond safely to any upcoming road turn. As 303 such, we suggest that two audio prompts be delivered when approaching a road turn: one 304 several hundred meters before the turn (serving a traditional navigational function) and 305 another approximately 1-2 s before the turn response is required (serving the significant 306 priming function that our results suggest). Indeed, this should have a cumulative priming 307 effect, similar to that observed in road sign studies using repetitive priming (e.g. Crundall \& 308 Underwood, 2001; Koyuncu \& Amado, 2008). Novel to the literature, our results suggest that 309 this will be especially effective when accompanied by a visual road sign instruction; priming 310 timing effects should also be taken into account in road sign placement.

311 In this experiment, we note differential effects for auditory primes compared to visual 312 primes or a multisensory combination of both. While all prime medium types elicit strong 313 priming effects, with congruent primes speeding responses to subsequent road scenes, 314 incongruent primes were less detrimental to performance when information was presented 315 aurally. Specifically, visual and audio-visual combination trials elicited classic priming 316 effects, with fastest responses to congruent trials, followed by control and incongruent trials. 317 For audio trials, a different pattern emerges. Again we see the fastest responses for congruent 318 trials, but incongruent trials elicit faster responses than control trials. This suggests that even 319 incorrect audio instruction can prime drivers to respond faster than a neutral phrase. This 320 wasn't the case for trials containing visual information, and we propose that this may be due 321 to the stronger visual ties between a road bend sign and a road bend, compared to the weaker 322 association between the words "turn left" and a left road turn. Additionally, up to one-third of 
323 people may experience mild difficulty when discriminating directional meaning from the 324 words "left" and "right" (McMonnies, 1990), making audio directional instructions a weaker 325 prompt.

326 Alternatively, the instruction to "turn" may provide a priming function in and of itself.

327 That is, the audio command to "turn left" may speed responses because it contains an 328 instruction to act (rather than serving a more descriptive "left turn" function). This might 329 explain why even incongruent audio information (containing the commands "turn left" or 330 "turn right") led to faster response times than control audio information ("hello"). 331 Interestingly, this does suggest that "getting it wrong" will be less detrimental to driving 332 performance for audio instructions compared to visual instructions.

334 driving studies (e.g. Lajunen et al., 1996), and it is considered useful to use lab-based work to 335 understand how drivers extract information from road sign cues (Castro \& Horberry, 2004). 336 Indeed, specific perceptual factors can be controlled and manipulated to a much greater 337 extent in a lab setting. As such, much research on the efficacy of road signs is laboratory338 based, where we can tightly control variables such as duration of presentation, size, colour 339 and contrast of the sign (Wogalter \& Laughery, 1996). In the case of this study, a lab-based 340 experiment facilitated a clean comparison of the relative priming efficacy of different types 341 of driving instruction. However, it is important to acknowledge that in a real-world driving 342 environment, information presented using an in-car device - whether serving a navigational 343 or priming purpose - will be competing for attentional demand with other visual and auditory 344 stimuli. In particular, driving requires that a high level of visual attention be paid to the road 345 scene, relative to auditory information. We should therefore be cautious in emphasising the 
346 superiority of visual priming over audio priming for road scenes on the basis of laboratory 347 findings.

In-car navigational systems traditionally only provide information when there is a navigational choice to be made. That is, a driver is only presented with visual or audio "turn 350 left" information when faced with a junction or motorway exit. The current study investigated 351 the advantages of audio, visual and multisensory priming where a different type of road 352 response is required - namely responding quickly and accurately to a road bend. Here, we 353 were investigating whether the priming function served by road signs indicating a bend in the 354 road ahead (e.g. Crundall \& Underwood, 2001) could be replicated using in-car systems. Our 355 results suggest that this should be the case. The response time advantages this type of priming 356 confers would likely be most useful in precision driving situations. For example, this type of 357 priming should be particularly useful in rally car driving, where extremely fast responses to 358 the road environment are required. The priming function served by presenting this type of 359 priming information - which is functionally different from navigational information currently 360 provided by in-car systems - may confer small advantages in normal driving scenarios as 361 well.

Responses in this experimental study were limited to button presses. In part, this design was chosen to control for extraneous perceptual variables in order to make a clean 364 comparison between visual, audio and multisensory road instruction primes, as mentioned 365 above. Another reason for this design choice was to closely replicate the conditions of 366 Crundall and Underwood's seminal study of the priming function of road signs (2001). In 367 doing so, we could test whether their findings of a priming effect of road signs might extend 368 to in-car audio cues as well, a hypothesis which has not before been tested. Based on our 369 findings, we recommend this as a promising line of investigation, and propose future 
370 simulator and real-world research to further investigate the priming potential of these audio 371 cues.

\section{Conclusion}

374 With the increased availability of in-car information systems, the question of how these 375 devices can best be used to prime drivers to respond, rather than simply provide navigational 376 information, is becoming more pertinent. Our experiment demonstrates the efficacy of audio 377 priming in responding to a road scene and suggests that the concurrent presentation of both 378 visual and audio primes should improve reaction speed in a driving setting. Overall, we 379 suggest a new research direction in the study of in-car information systems focusing on the 380 priming potential of these devices.

\section{References}

Agent, K. R., Stamatiadis, N., \& Jones, S. (1996). Development of Accident Reduction Factors. Report No. KTC-96-13. Lexington, KY: University of Kentucky.

Barrow, K. (1991). Human factors issues surrounding the implementation of in-vehicle navigation and information systems. SAE Technical Paper Series 910870, 12431257. Warrendale, PA: Society of Automobile Engineers. Doi: 10.4271/910870. 
391 Burnett, G. E. (2000). Turn right at the traffic lights: The requirement for landmarks in 392 vehicle navigation systems. The Journal of Navigation, 53, 499-510. Doi: $10.1017 / \mathrm{s} 0373463300001028$.

Burns, P. C. (1997). Navigation and the Ageing Driver. Unpublished PhD Dissertation. 395 Loughborough University Institutional Repository, Loughborough, UK.

396 397 398
Broy, M. (2006). Challenges in Automotive Software Engineering. Proceedings of the $28^{\text {th }}$ international conference on software engineering, 33-42. Doi: $10.1145 / 1134285.1134292$.

Castro, C., \& Horberry, T. (2004). The Human Factors of Transport Signs. London: CRC Press. Doi: 10.1201/9780203457412.

Charlton, S. G. (2006). Conspicuity, memorability, comprehension and priming in road hazard warning signs. Accident Analysis and Prevention, 38, 496-506. Doi: 10.1016/j.aap.2005.11.007.

Creasy, T., \& Agent, K. R. (1985). Development of Accident Reduction Factors. Report No. UKTRP-85-6. Lexington, KY: University of Kentucky.

Crundall, D., \& Underwood, G. (2001). The priming function of road signs. Transportation Research Part F 4, 187-200. Doi: 10.1016/S1369-8478(01)00023-7.

Dalton, P., Agarwal, P., Fraenkel, N., Baichoo, J., \& Masry, A. (2013). Driving with navigational instructions: Investigating user behaviour and performance. Accident Analysis and Prevention, 50, 298-303. Doi: 10.1016/j.aap.2012.05.002.

Department for Transport (2007). Highway Code: traffic signs publication, Fifth Edition. London: Crown. 
413 Dingus, T., McGehee, D., Hulse, M., Jahns, S., \& Manakkal, N. (1995). Travtrek evaluation 414 task C3 - Camera Car study. Report No. FHWA-RD-94-076. Office of Safety and 415

416 Fastenmeier, W., Haller, R., \& Lerner, G. (1994). A preliminary safety evaluation of route 417 guidance comparing different MMI concepts. Proceedings of First World Congress on Applications of Transport Telematics and Intelligent Vehicle Highway Systems,

420 Fisher, J. (1992). Testing the effect of road traffic signs' informational value on driver behaviour. Human Factors, 34, 231-237. Doi: 10.1177/001872089203400208. $4,1750-1756$.

Ho, C., Reed N., \& Spence, C. (2007). Multisensory in-car warning signals for collision avoidance. Human Factors, 46, 1107-14. Doi: 10.1518/001872007x249965. Proceedings of the Human Factors and Ergonomics Society Annual Meeting, 39, 1072-1076. Doi: 10.1177/154193129503901702.

Huber, D. E., \& O’Reilly, R. C. (2003). Persistence and accommodation in short-term priming and other perceptual paradigms: temporal segregation through synaptic depression. Cognitive Science, 27, 403-430. Doi: 10.1207/s15516709cog2703_4.

Jensen, B. S., Skov, M. B., \& Thiruravichandran, N. (2010). Studying driver attention and behaviour for three configurations of GPS navigation in real traffic driving. Proceedings of the SIGCHI Conference on Human Factors in Computing Systems, 1271-1280. Doi: 10.1145/1753326.1753517. 
434 Johansson, G., \& Backlund, F. (1970). Drivers and road signs. Ergonomics, 13, 749-759.

$435 \quad$ Doi: $10.1080 / 00140137008931202$.

436 Kinchla, R. (1974). Detecting target elements in multielement arrays: A confusability model.

437 Perception and Psychophysics, 15, 149-158.

438 Klauer, S. G., Guo, F., Sudweeks, J., \& Dingus, T. A. (2010). An analysis of driver

439 inattention using a case-crossover approach on 100-car data: Final report. Report

440 No. DTNH22-00-C-07007. Washington, DC: Office of Human-Vehicle

441 Performance Research. Doi: 10.1037/e729232011-001.

442 Koyuncu, M., \& Amado, S. (2008). Effects of stimulus type, duration and location on

443 priming of road signs: Implications for driving. Transportation Research Part F II,

444 108-125. Doi: 10.1016/j.trf.2007.08.005.

445 Lajunen, T., Hakkarainen, P., \& Summala, H. (1996). The ergonomics of road signs: Explicit

446 and embedded speed limits. Ergonomics, 39(8), 1069-1083. Doi:

$447 \quad 10.1080 / 00140139608964528$.

448 Labaile, G. (1990). In-car road information: Comparisons of auditory and visual presentation.

449 Proceedings of the Human Factors and Ergonomics Society, 34, 623-627. Doi:

$450 \quad 10.1177 / 1541931290034909$.

451 Liang, Y., Lee, J. D., \& Yekhshatyan, L. (2012). How dangerous is looking away from the 452 road? Algorithms predict crash risk from glance patterns in naturalistic driving.

453 Human Factors, 54, 1104-1116. Doi: 10.1177/0018720812446965. 
454 Liu, Y.-C. (2001). Comparative study of the effects of auditory, visual and multimodality 455 displays on drivers' performance in advanced traveller information systems. 456 Ergonomics 44, 425-442. Doi: 10.1080/0014030010011369.

457 McMonnies, C. W. (1990). Left-right discrimination in adults. Clinical and Experimental $458 \quad$ Optometry, 73, 155-158. Doi: 10.1111/j.1444-0938.1990.tb03116.x.

459 Miller, J. (1982). Divided attention: Evidence for coactivation with redundant signals. 460 Cognitive Psychology, 14, 247-279. Doi: 10.1016/0010-0285(82)90010-X.

461 Milošević, S., \& Gajić, R. (1986). Presentation factors and driver characteristics affecting 462 road sign registration. Ergonomics, 29, 807-815. Doi:

$463 \quad 10.1080 / 00140138608968317$.

464 Mori, M., \& Abdel-Halim, M. H. (1981). Road sign recognition and non-recognition.

465 Accident Analysis and Prevention, 13, 101-115. Doi: 10.1016/0001-

$466 \quad 4575(81) 90023-3$.

467 Raab, D. (1962). Statistical facilitation of simple reaction times. Transactions of the New $468 \quad$ York Academy of Sciences, 24, 574-590.

469 Ratcliff, R. (1993). Methods for dealing with reaction time outliers. Psychological Bulletin, $470 \quad 114,510-532$. Doi: 10.1037/0033-2909.114.3.510.

471 Regan, M. A., Oxley, J. A., Godley, S. T., \& Tingvall, C. (2001). Intelligent transport system: 472 Safety and human factors issues. Report No. 01/01. Victoria, Australia: Royal $473 \quad$ Automobile Club.

474 Simons-Morton, B. G., Guo, F., Klauer, S. G., Ehsani, J. P., \& Pradhan, A. K. (2014). Keep 475 your eyes on the road: Young driver crash risk increases according to duration of 
476

477

478

479

480

481

482

483

484

485

486

487

488

489

490

491

492

493

494

495

496

497

distraction. Journal of Adolescent Health, 54, S61-S67. Doi:

10.1016/j.adohealth.2013.11.021.

Spence, C., \& Santangelo, V. (2009). Capturing spatial attention with multisensory cues: A review. Hearing Research, 258, 134-142. Doi: 10.1016/j.heares.2009.04.015.

Srinivasan, R., \& Jovanis, P. P. (1997). Effect of in-vehicle route guidance systems on driver workload and choice of vehicle speed: Findings from a driving simulator experiment. In Y. I. Noy (Ed.). Ergonomics and safety of intelligent driver interfaces, 97-114. New Jersey: Lawrence Erlbaum Associates, Publishers.

Summala, H., \& Hietamäki, J. (1984). Drivers’ immediate responses to traffic signs. Ergonomics 27, 205-216. Doi: 10.1080/0014038408963478.

Walker, J., Alicandri, E., Sedney, C., \& Roberts, K. (1991). In-vehicle navigation devices: Effects on the safety of driver performance. Vehicle Navigation and Information Systems Conference, 2, 499-525. Doi: 10.1109/VNIS.1991.205796.

Wogalter, M.S., \& Laughery, K.R. (1996). Warning sign and label effectiveness. Current Directions in Psychological Science, 5(2), 33-37.

Wu, C.F., Huang, W.F., \& Wu, T.C. (2009). A study on the design of voice navigation of car navigation system. Proceedings of the 13th International Conference on HumanComputer Interaction. Ambient, Ubiquitous and Intelligent Interaction.Part III. ISBN 978-3-642-02580-8.

Xie, C., Zhu, T., Guo, C., \& Zhang, Y. (2013). Measuring IVIS impact to driver by on-road test and simulator experience. Procedia - Social and Behavioural Sciences, 96, 1566-1577. Doi: 10.1016/j.sbspro.2013.08.178. 
498

499

500

501

502

503

504

505

506

507

508 Figure Captions

509 Figure 1: Sequence of trial events. Primes were either visual (road sign), audio (computerised

510 voice), or both (as illustrated in this example).

511

512 Figure 2: Mean response times to correctly respond to a road turn when the prime stimulus

513 was congruent (diagonal lines) or incongruent (checker) to the target road turn direction, or a

514 control (dots). Error bars represent the standard error of the mean. 
516 Figure 3: Mean response times to correctly respond to a road turn when the prime stimulus

517 was multisensory (solid grey), visual (vertical lines) or audio (horizontal lines). Error bars 518 represent the standard error of the mean.

519

520 Figure 4: Mean response times to correctly respond to a road turn following a visual prime, 521 multisensory prime or an audio prime when the prime stimulus was congruent (diagonal 522 lines) or incongruent (checker) to the target road turn direction, or a control (dots). Error bars 523 represent the standard error of the mean. 


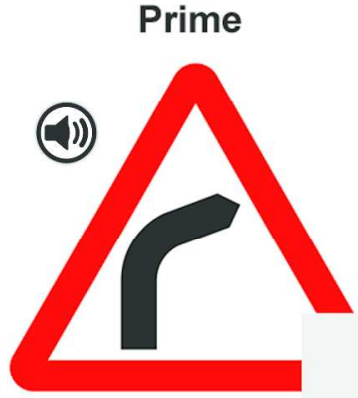

Fixation

$882 \mathrm{~ms}$

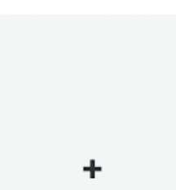

$700-1200 \mathrm{~ms}$

Figure 1: Sequence of trial events. Primes were either visual (road sign), audio (computerised voice), or both (as illustrated in this example).

$364 \times 346 \mathrm{~mm}(300 \times 300$ DPI) 


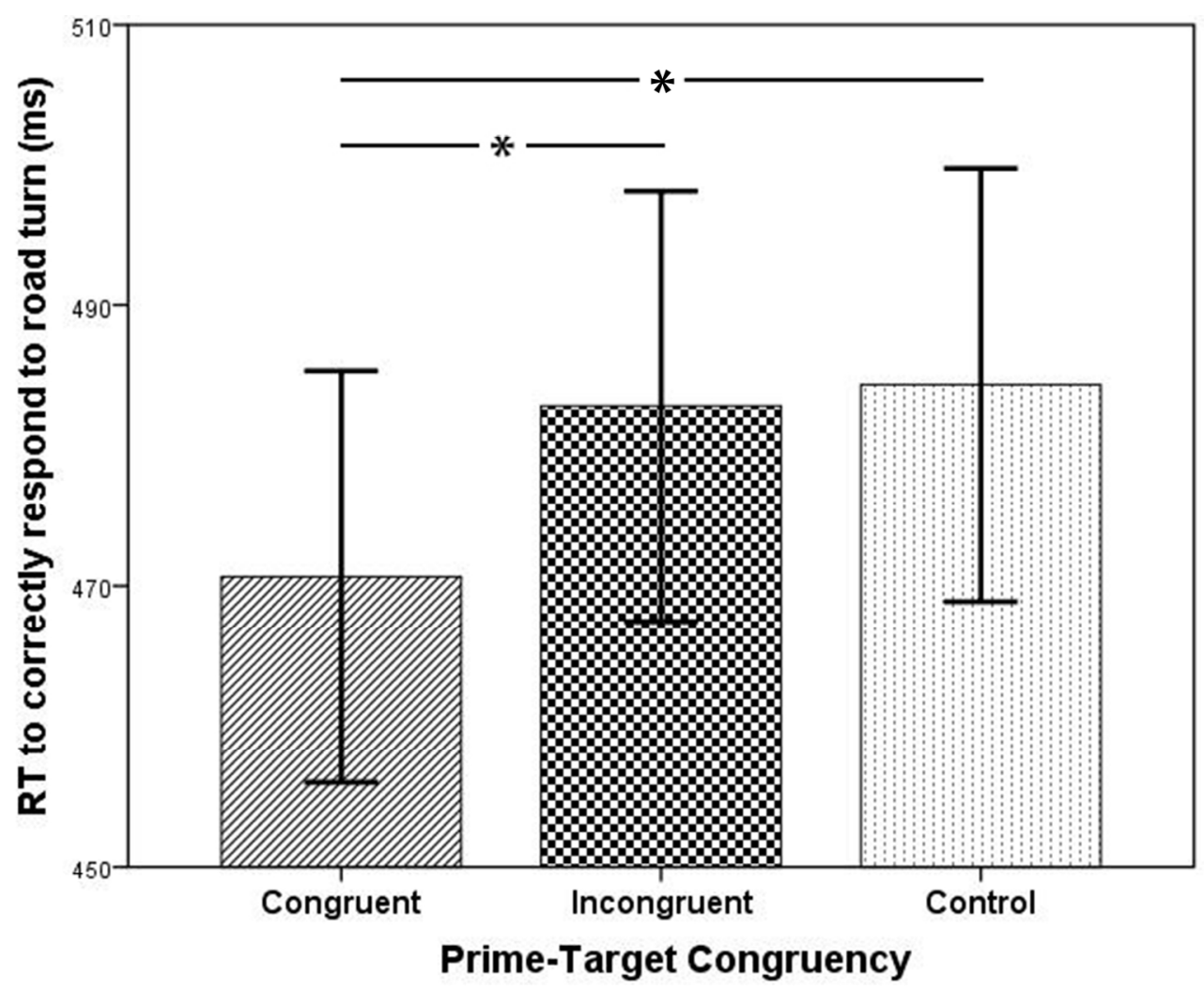

Figure 2: Mean response times to correctly respond to a road turn when the prime stimulus was congruent (diagonal lines) or incongruent (checker) to the target road turn direction, or a control (dots). Error bars represent the standard error of the mean.

$176 \times 141 \mathrm{~mm}(300 \times 300$ DPI $)$ 


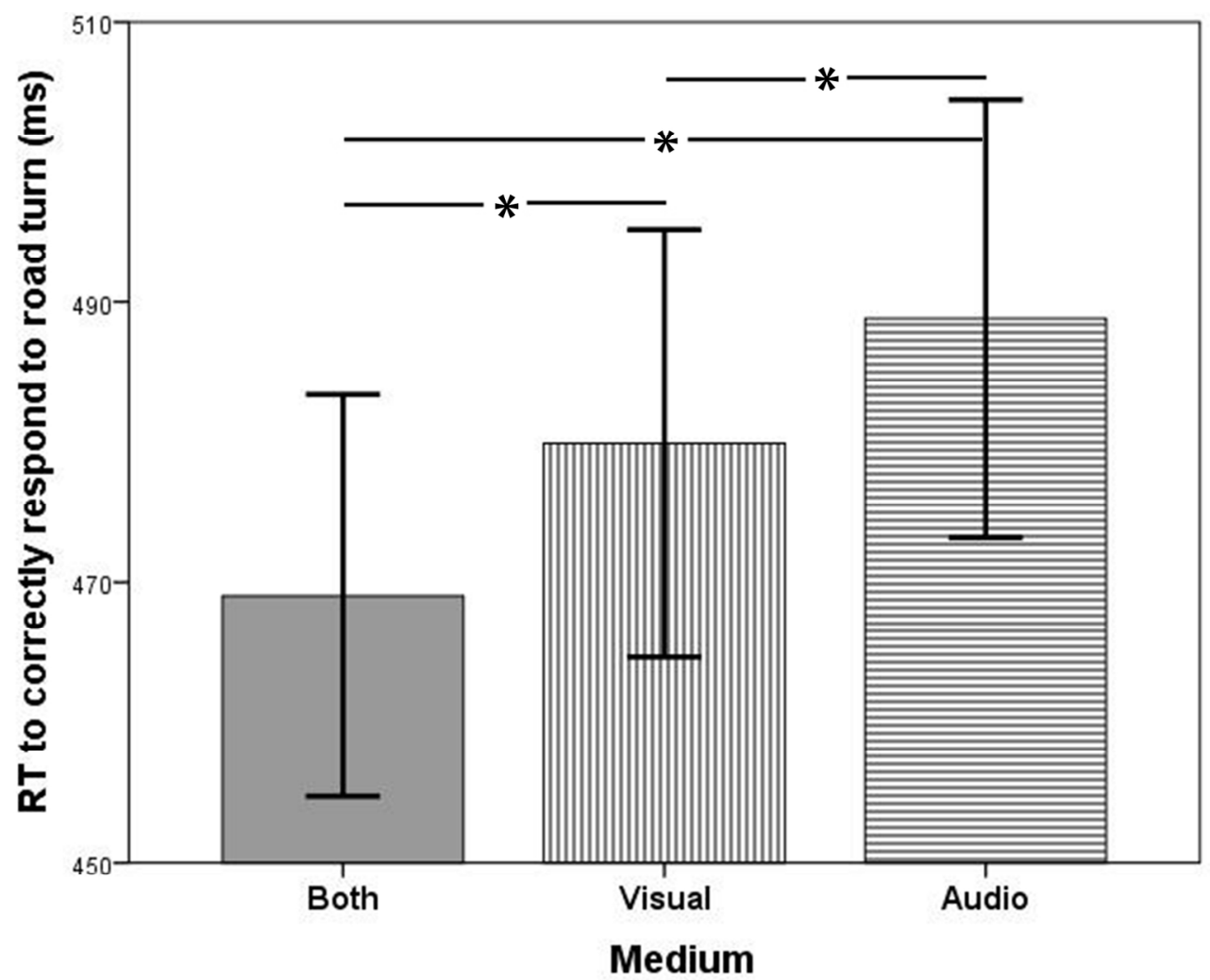

Figure 3: Mean response times to correctly respond to a road turn when the prime stimulus was multisensory (solid grey), visual (vertical lines) or audio (horizontal lines). Error bars represent the standard error of the mean.

$176 \times 141 \mathrm{~mm}$ ( $300 \times 300$ DPI) 


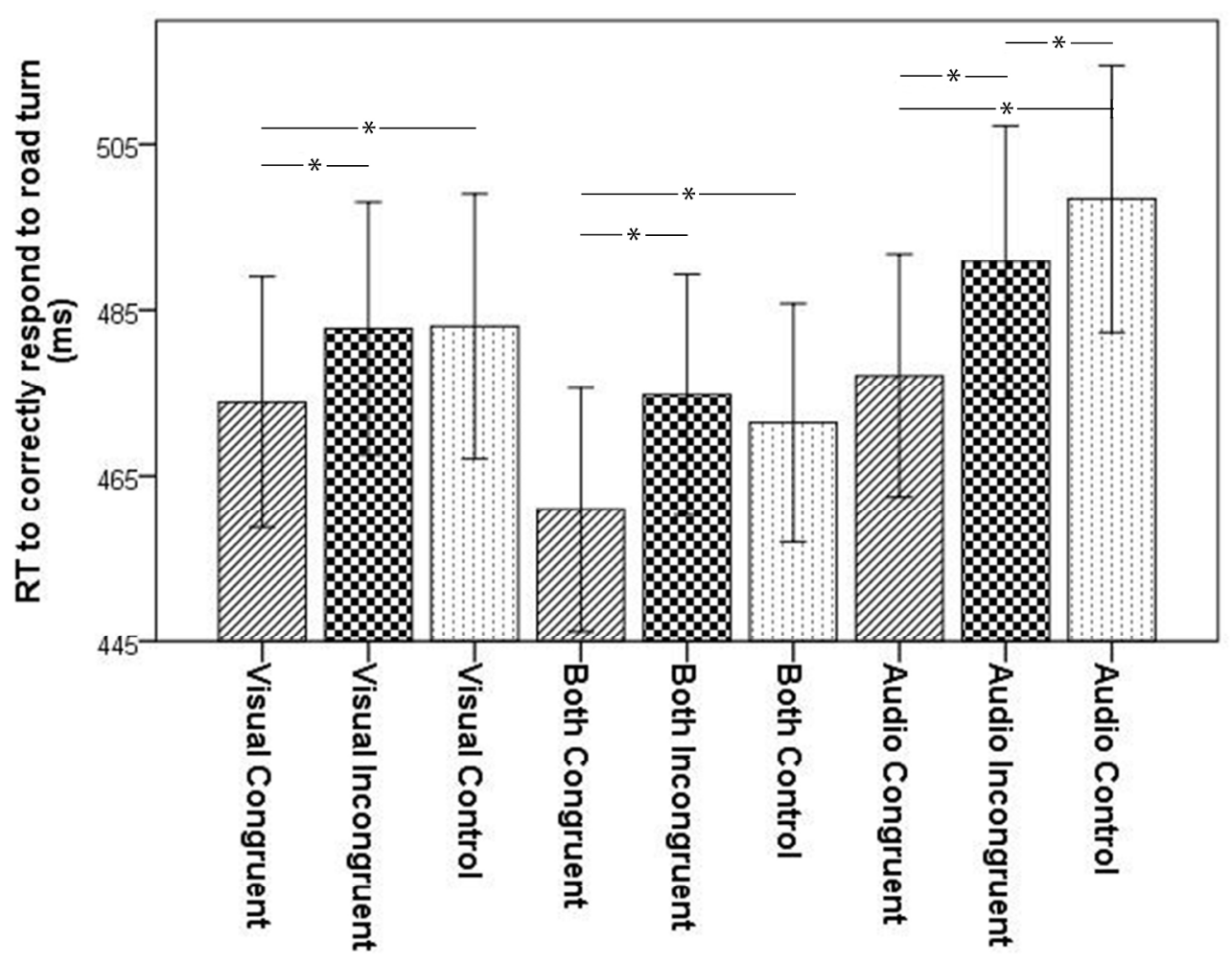

Figure 4: Mean response times to correctly respond to a road turn following a visual prime, multisensory prime or an audio prime when the prime stimulus was congruent (diagonal lines) or incongruent (checker) to the target road turn direction, or a control (dots). Error bars represent the standard error of the mean. 